\title{
Non-invasive and micro-invasive treatments to arrest active occlusal carious lesions in erupting permanent molars: A randomized clinical trial
}

Jonas Almeida RODRIGUES(a)

Nicole Marchioro SANTOS(a)

Claudia Brito AZEVEDO(a)

Alex Nogueira HAAS(b)

Tathiane Larissa LENZI (a) (iD

(a) Universidade Federal do Rio Grande do Sul - UFRGS, School of Dentistry, Department of Surgery and Orthopedics, Porto Alegre, RS, Brazil.

(b) Universidade Federal do Rio Grande do Sul - UFRGS, School of Dentistry, Department of Conservative Dentistry, Porto Alegre, RS, Brazil.
Declaration of Interests: The authors certify that they have no commercial or associative interest that represents a conflict of interest in connection with the manuscript.

Corresponding Author:

Jonas Almeida Rodrigues

Email: jorodrigues@ufrgs.br

ht1ps://doi.org/10.1590/1807-3107bor-2021.vol35.0058

Submitted: May 9, 2020

Accepted for publication: November 25, 2020

Last revision: January 8, 2021
Abstract: This study aimed to evaluate the efficacy of non-invasive and micro-invasive treatments on the arrest of occlusal enamel carious lesions in erupting permanent molars. This two-arm randomized clinical trial included 27 subjects, aged 5-11 years, with 64 erupting permanent molars presenting active occlusal enamel carious lesions (as assessed by the International Caries Detection and Assessment System [ICDAS]; scores 1-3). The sample was randomly assigned into two treatment groups: 1) resin-modified glass ionomer cement sealant (Clinpro XT Varnish; 3M ESPE) and 2) 4-week topical fluoride varnish application (Duraphat; Colgate). All children and parents received oral hygiene and dietary instructions. Teeth were evaluated at baseline and 3, 6, 9, and 12 months regarding the eruption stage, biofilm accumulation, as well as severity and activity of the carious lesions. The Kaplan-Meier method was used to evaluate the survival estimates for inactivation of the carious lesions for both treatment groups. Multivariate Cox regression models with shared frailty were performed to identify factors associated with the outcome $(p<0.05)$. After 12 months, 22\% and 3\% of the lesions treated with topical fluoride varnish and sealant, respectively remained active. The adjusted model demonstrated that younger children had a higher probability of active enamel carious lesions arresting (hazard ratio [HR] 0.42, 95\% confidence interval $[\mathrm{CI}]$ 0.22-0.78; $\mathrm{p}=0.01$ ). However, the probability of active enamel carious lesions arresting after sealant application was 8.85 times higher compared with fluoride varnish applications $(p=0.01)$. Sealing is a more effective approach than fluoride varnish for arresting occlusal enamel carious lesions in erupting permanent molars.

Keywords: Tooth Eruption; Dental Caries; Fluorides; Glass Ionomer Cements.

\section{Introduction}

Despite the decline in the prevalence and progression rate of caries in children and adolescents worldwide, ${ }^{1}$ permanent molars continue to be the most frequently decayed teeth. ${ }^{2}$ The eruption stage is a predictor of caries on permanent teeth. ${ }^{3}$ The average period of eruption is approximately 
15-27 months for the primary and secondary permanent molars, respectively. ${ }^{4}$ The difficult access to molars, presence of gingival tissue on the occlusal surface, and absence of full occlusion are contributing factors for biofilm accumulation and, subsequently, caries activity. ${ }^{5}$ Most occlusal carious lesions tend to arrest/revert when permanent molars reach the occlusal plane; however, a significant proportion of these lesions remain active. ${ }^{5}$ This situation highlights the need for early caries assessment of these molar surfaces to establish minimally invasive strategies for arresting active enamel carious lesions at the initial eruption stages.

Long-term, individualized, and non-invasive treatment based on patient education and professional acidulated phosphate fluoride gel applications displayed promising results in controlling occlusal carious lesions in erupting first permanent molars. ${ }^{6}$ Recently, it was demonstrated that a standardized treatment protocol comprising 4 -week topical fluoride gel application and oral hygiene instructions, was not adequate for caries control in partially erupted permanent molars.?

There is moderate evidence for the use of sealants plus fluoride varnish (3-6 monthly applications), or sealants alone, over fluoride varnish alone (3-6 monthly applications) to arrest or reverse noncavitated carious lesions on the occlusal surface of permanent teeth. ${ }^{8}$ However, no previous study has compared the efficacy of both strategies in controlling enamel carious lesions on the occlusal surface of erupting permanent molars. Therefore, this randomized clinical trial investigated the efficacy of a resin-modified glass ionomer cement sealant and a 4-week topical fluoride varnish application protocol on arresting active occlusal enamel carious lesions of the erupting permanent molars.

\section{Methodology}

\section{Study design and ethical concern}

This was a two-arm, parallel, randomized clinical trial that followed the Consolidated Standards of Reporting Trials statement. ${ }^{9}$ The local Ethics Committee on Investigations Involving Human Subjects of the Federal University of Rio Grande do
Sul reviewed and approved the protocol and consent form for this study (CAAE 31409014.8.0000.5347). Written informed consent was obtained from the guardians of the participants before treatment initiation (REBEC Register Number: RBR-32NYHT). The study was conducted at the Children and Youth Dental Clinic, School of Dentistry, Federal University of Rio Grande do Sul (UFRGS), Porto Alegre, Brazil, from June 2014 to June 2015.

\section{Sample calculation}

For calculation of the sample size, a reduction in caries incidence in permanent molars treated with sealant and fluoride varnish was considered, totaling $64.5 \%$ and $27.3 \%,{ }^{10}$ respectively. Therefore, considering a significance level of 0.05 , a power of 0.80 , and using a one-tailed test for non-inferiority studies $(\mathrm{d}=-0.10)$, a minimum of 12 children per group is indicated. Considering a $10 \%$ potential sample loss, a total of 14 children per group were recruited, resulting in a study sample of 28 children.

\section{Sample selection}

A total of 102 children (aged 5-11 years) were examined by an experienced dentist (J.A.R.), who checked the inclusion and exclusion criteria (Figure 1). The participants were recruited in the order in which they attended the screening appointment. The clinical evaluations were performed under lighting using a plain mouth mirror and ballpoint probe (Hu-Friedy Manufacturing Co., Chicago, USA) after the teeth had been cleaned, dried, and isolated. Children presenting with good general health and cooperative behavior and who completed the clinical procedure with a minimum of one erupting permanent molar with active enamel carious lesions (assessed by the International Caries Detection and Assessment System [ICDAS]; scores 1-3) ${ }^{11,12}$ on the occlusal surface, were included in this study. Activity status was assessed based on the clinical appearance of the occlusal surfaces including brightness, color, and texture. ${ }^{13}$ Teeth with occlusal surfaces completely covered by the gingival tissue, and teeth with hypoplastic defects, restorations, or sealants, were not included in the sample. Participants requiring any other dental treatment were referred to the Dental School of the 
University. All children and their guardians/parents received general information about oral hygiene and dietary habits for the prevention and control of dental caries. Based on the pre-established criteria, 27 volunteers were selected for this study (Figure 1).

\section{Training and calibration}

An examiner (J.A.R.) underwent $8 \mathrm{~h}$ of specific training involving theoretical explanations, discussion, and assessment of representative photographs of the criteria used in this study for evaluation of the eruption stage ${ }^{12}$, biofilm accumulation ${ }^{12}$, severity ${ }^{11}$, and activity ${ }^{13}$ of the carious lesions. The training session also included the clinical examination of 10 patients who did not participate in the study. First, the permanent molars were classified according to the type (first or second) and eruption stage as follows: a) partially erupted occlusal surface; b) fully erupted occlusal surface, with more than half of the buccal surface of the tooth covered with gingival tissue; c) fully erupted occlusal surface, with less than half of the buccal surface of the tooth covered with gingival tissue; and d) full occlusion. ${ }^{12}$ Subsequently, biofilm accumulation on occlusal surfaces was evaluated as follows: a) absence of visible biofilm, b) hardly detectable biofilm in pits and fissures, c) easily detectable biofilm in pits and fissures, and (4) occlusal surface partially or completely covered by biofilm. ${ }^{12}$

After professional prophylaxis, the occlusal surfaces were classified according to the severity of the carious lesions using ICDAS, ${ }_{1}^{11}$ and activity status was assessed based on the clinical appearance of the occlusal surfaces including brightness, color, and texture. ${ }^{13}$ The ballpoint probe (Hu-Friedy Manufacturing Co., Chicago, USA) was used for the evaluation of surface texture (smooth or rough). Moreover, the lesions were classified as active (white, opaque, and/or rough enamel) or inactive (white or brownish but shiny and/or hard/smooth).

For determining the intra-examiner agreement, evaluations were repeated after $1 \mathrm{~h}$ for biofilm accumulation and after 1 week for the other criteria. For all criteria, the intra-examiner kappa values were higher than 0.80 .



Figure 1. Flow diagram of the study. 


\section{Randomization, allocation, and blinding}

Randomization was performed by a staff member (A.N.H.) who was not involved in any of the clinical trial phases. Children were randomly allocated to each group according to a sequence obtained using the appropriate software (Random Allocation 1.0, Isfahan, Iran). When the same patient had more than one tooth, all teeth received the same treatment. The allocation concealment was guaranteed by the use of opaque and numbered individual envelopes. The operator, participants, and examiner could not be blinded.

\section{Intervention}

Two treatment groups were defined: topical fluoride varnish application (Duraphat; Colgate, São Paulo, SP, Brazil) and the use of resin-modified glass ionomer cement sealant (Clinpro XT Varnish; 3M ESPE, St. Paul, SA). Both products were applied according to the manufacturers' instructions after professional prophylaxis using a Robson brush, pumice, and water, and under relative isolation using a suction tube and cotton rolls. No difficulties were observed during tooth isolation.

Occlusal surfaces were air-dried for $5 \mathrm{~s}$, and a thin layer of fluoride varnish was applied over the active carious lesion on the entire occlusal surface using a cotton applicator. The participants were instructed not to eat solid foods for a minimum of $1 \mathrm{~h}$ after the procedure, and not to brush their teeth in the following $4 \mathrm{~h}$. A protocol of four professional applications of varnish fluoride at weekly intervals was performed. ${ }^{14}$

For sealant application, occlusal surfaces were acidetched with 35\% phosphoric acid gel (Etchant Scotch Bond, 3M ESPE, St. Paul, USA) for $15 \mathrm{~s}$, rinsed with water for $30 \mathrm{~s}$, and air-dried for $5 \mathrm{~s}$. The paste/liquid components were mixed for $15 \mathrm{~s}$, and a thin layer of the material was applied to the tooth surface, followed by light curing (Radii-cal; SDI, Victoria, Melbourne, AUS) for $20 \mathrm{~s}$.

In both groups, the children and parents received special instructions on dietary habits and appropriate techniques for plaque control on the occlusal surface of erupting permanent molars. The toothbrush was held in the buccolingual direction, with the bristles facing toward the occlusal surface, and moved in small circular motions..$^{15}$ It was recommended that the parents assist their children up to the age of 7 or 8 years to brush their teeth with $1100 \mathrm{ppm}$ fluoridated toothpaste at least once daily. A single experienced dentist (C.B.A.) performed all treatments.

\section{Assessments}

A calibrated examiner (J. A. R) evaluated the occlusal surfaces of erupting permanent molars after 3, 6, 9, and 12 months of treatment. Eruption stage,$^{12}$ biofilm accumulation, ${ }^{12}$ severity, ${ }^{11}$ and activity $^{13}$ of the carious lesions were recorded. The patients were recalled for follow-up telephonically, and the evaluations were performed on a dental chair using artificial dental light, a triple syringe, a plain mouth mirror, and a probe. A drawing of each evaluated occlusal surface was made to record the location of carious lesions more accurately and to allow future comparisons during clinical follow-up periods. Sealed teeth only had the visible part of the occlusal surface re-examined in the follow-up evaluations. Therefore, only occlusal sites (bottoms and walls of pits and fissures) in which sealants had been lost or newly erupted parts of the occlusal surfaces could be evaluated. Sealant retention was assessed as follows: total retention of sealant on the occlusal surface or the partial or complete absence of the sealant on the occlusal surface. The outcome measure was carious lesion arrest. Active enamel lesions in teeth treated with fluoride varnish were assessed for caries activity status, based on the clinical appearance of the occlusal surfaces including brightness, color, and texture, as described above ${ }^{13}$. Arrest of the carious lesions in the sealed occlusal surfaces was confirmed when no demineralization was observed adjacent to the sealant, or when no active lesion was observed after partial or total loss of the sealant.

\section{Statistical analysis}

Data analyses were performed using STATA software 12.0 (Stata Corp., College Station, Texas, USA). The descriptive analysis provided the distribution summary according to the independent variables: 
age, sex, eruption stage, biofilm accumulation, and type of treatment. The primary outcome of the present study was the arrest of active carious lesions. The Kaplan-Meier method was used to evaluate the survival estimates for inactivation of the carious lesions for both treatments.

Multivariate Cox regression models with shared frailty were performed to identify factors associated with the outcome. These models consider that observations within the same group (the patient) are correlated, sharing the same frailty, and are analogous to multilevel regression models with random effects. A test of the proportional hazards assumption was performed for each independent variable before including them in the regression analysis. Hazard ratios (HRs) and their respective 95\% confidence intervals (CIs) were also obtained. A backward stepwise procedure was used to select covariates in the fitting of the model. Only variables with $p<0.20$ were selected for inclusion in the final model. The significance level was set at $5 \%$.

\section{Results}

The sample comprised 64 erupting permanent molars (58 first and 6 second molars; 21 with ICDAS score 1, 32 with ICDAS score 2, and 11 with ICDAS score 3 ) of 27 children. Thirty-three teeth (mean of $2.4 \pm 1.5$ teeth per child) of 13 children (11 females and 2 males), with a mean age of $7.5 \pm 1.6$ years, were sealed. Thirty-one molars (mean $2.3 \pm 1.2$ teeth per child) of 14 children ( 8 females and 6 males), with a mean age of $8.4 \pm 1.6$ years, received fluoride varnish applications. There were no dropouts after the follow-up (Figure 1) and Tables 1 and 2 summarizes the sample characteristics. The participants' oral hygiene had a subtle improvement when comparing biofilm accumulation data at baseline and after 12 months, irrespective of the treatment type. After 12 months, $93.5 \%$ of teeth that received varnish application and $78.8 \%$ of sealed molars reached full occlusion.

Figure 2 illustrates the Kaplan-Meier survival curves according to the treatments. Furthermore, enamel carious lesions that received a fluoride varnish application were more likely to remain active for longer periods. After 12 months, $78 \%$ of the carious lesions treated with topical fluoride varnish applications were arrested, whereas $97 \%$ of the sealed lesions were arrested $(p=0.04)$. Seven lesions $(22 \%)$ remained active after fluoride varnish applications, while the majority (four lesions) progressed to enamel breakdown (ICDAS score $=3$ ). Moreover, 28 sealants had total retention, four sealants were partially lost, one sealant was completely lost, and a carious lesion was detected ( $3 \%$ of failure).

Table 3 illustrates the unadjusted and adjusted HR for failures according to the independent variables. The adjusted model demonstrated that younger children had a higher probability of arresting active enamel carious lesions (HR 0.42, 95\%CI 0.22-0.78; $\mathrm{p}=0.01$ ). The likelihood of active enamel carious lesions to arrest after sealant application was 8.85 times higher than that of fluoride varnish applications (HR 8.85 95\%CI 1.57-49.78; $\mathrm{p}=0.01$ ).

Table 1. Sample distribution at baseline.

\begin{tabular}{|c|c|c|}
\hline \multirow{2}{*}{ Variables } & Fluoride varnish & Sealant \\
\hline & $(n=31)$ & $(n=33)$ \\
\hline Age (years $\pm S D$ ) & $8.32( \pm 1.77)$ & $6.87( \pm 0.92)$ \\
\hline \multicolumn{3}{|l|}{ Gender } \\
\hline Male $(\mathrm{n}=8)$ & 6 & 2 \\
\hline Female $(n=19)$ & 8 & 11 \\
\hline \multicolumn{3}{|l|}{ Biofilm accumulation } \\
\hline 1 & 0 & 0 \\
\hline 2 & 3 & 2 \\
\hline 3 & 13 & 19 \\
\hline 4 & 15 & 12 \\
\hline \multicolumn{3}{|l|}{ Eruption stage } \\
\hline 2 & 10 & 8 \\
\hline 3 & 21 & 25 \\
\hline \multicolumn{3}{|l|}{ ICDAS } \\
\hline 1 & 8 & 13 \\
\hline 2 & 19 & 13 \\
\hline 3 & 4 & 7 \\
\hline \multicolumn{3}{|l|}{ Type of molar } \\
\hline First & 26 & 32 \\
\hline Second & 5 & 1 \\
\hline \multicolumn{3}{|l|}{ Dental Arch } \\
\hline Upper & 16 & 18 \\
\hline Lower & 15 & 15 \\
\hline
\end{tabular}

SD: standard deviation; ICDAS: International caries detection and assessment system 
Non-invasive and micro-invasive treatments to arrest active occlusal carious lesions in erupting permanent molars: a randomized clinical trial

Table 2. Sample description according to treatments at baseline and follow-up periods.

\begin{tabular}{|c|c|c|c|c|c|c|c|c|c|c|}
\hline \multirow{2}{*}{ Variables } & \multicolumn{2}{|c|}{ Baseline } & \multicolumn{2}{|c|}{3 months } & \multicolumn{2}{|c|}{6 months } & \multicolumn{2}{|c|}{9 months } & \multicolumn{2}{|c|}{12 months } \\
\hline & Sealant & $\begin{array}{c}\text { Fluoride } \\
\text { varnish }\end{array}$ & Sealant & $\begin{array}{l}\text { Fluoride } \\
\text { varnish }\end{array}$ & Sealant & $\begin{array}{c}\text { Fluoride } \\
\text { varnish }\end{array}$ & Sealant & $\begin{array}{l}\text { Fluoride } \\
\text { varnish }\end{array}$ & Sealant & $\begin{array}{l}\text { Fluoride } \\
\text { varnish }\end{array}$ \\
\hline \multicolumn{11}{|c|}{ Biofilm accumulation } \\
\hline \multirow{2}{*}{1} & 0 & 0 & 2 & 3 & 4 & 3 & 6 & 6 & 3 & 6 \\
\hline & $0 \%$ & $0 \%$ & (6.1\%) & $(9.7 \%)$ & (12.1\%) & $(9.7 \%)$ & (18.2\%) & (19.4\%) & (9.1\%) & (19.4\%) \\
\hline \multirow{2}{*}{2} & 2 & 3 & 9 & 9 & 15 & 10 & 9 & 12 & 14 & 13 \\
\hline & (6.1\%) & $(9.7 \%)$ & $(27.3 \%)$ & $(29.0 \%)$ & (45.5\%) & (32.3\%) & $(27.3 \%)$ & $(38.7 \%)$ & (42.4\%) & (41.9\%) \\
\hline \multirow{2}{*}{3} & 19 & 13 & 18 & 9 & 9 & 13 & 17 & 9 & 10 & 10 \\
\hline & (57.0\%) & (41.9\%) & $(54.5 \%)$ & $(29.0 \%)$ & $(27.3 \%)$ & (41.9\%) & (51.5\%) & $(29.0 \%)$ & (30.3\%) & (32.3\%) \\
\hline \multirow{2}{*}{4} & 12 & 15 & 4 & 10 & 5 & 5 & 1 & 4 & 6 & 2 \\
\hline & $(36.4 \%)$ & $(48.4 \%)$ & (12.1\%) & (32.3\%) & (15.2\%) & (16.6\%) & (3.0\%) & (12.9\%) & (18.2\%) & (6.5\%) \\
\hline \multicolumn{11}{|c|}{ Eruption stage } \\
\hline \multirow{2}{*}{2} & 8 & 10 & 3 & 8 & 2 & 2 & 0 & 0 & 0 & 0 \\
\hline & (24.2\%) & (32.3\%) & (9.1\%) & (25.8\%) & (6.1\%) & (6.5\%) & $0 \%$ & $0 \%$ & $0 \%$ & $0 \%$ \\
\hline \multirow{2}{*}{3} & 25 & 21 & 19 & 10 & 14 & 9 & 11 & 9 & 7 & 2 \\
\hline & (75.7\%) & (67.7\%) & (57.6\%) & (32.3\%) & (42.4\%) & (29.0\%) & (33.3\%) & (29.0\%) & (21.2\%) & (6.5\%) \\
\hline \multirow{2}{*}{4} & 0 & 0 & 11 & 13 & 17 & 20 & 22 & 22 & 26 & 29 \\
\hline & $0 \%$ & $0 \%$ & (33.3\%) & (41.9\%) & (51.5\%) & (64.5\%) & $(66.7 \%)$ & (71.0\%) & (78.8\%) & (93.5\%) \\
\hline
\end{tabular}

Eruption stage: (1) partially erupted occlusal surface; (2) fully erupted occlusal surface, with more than half of the buccal surface of the tooth covered with the gingival tissue; (3) fully erupted occlusal surface; and (4) full occlusion. Biofilm accumulation: (1) absence of visible biofilm; (2) hardly detectable biofilm in pits and fissures; (3) easily detectable biofilm in pits and fissures; and (4) occlusal surface partially or completely covered by biofilm.

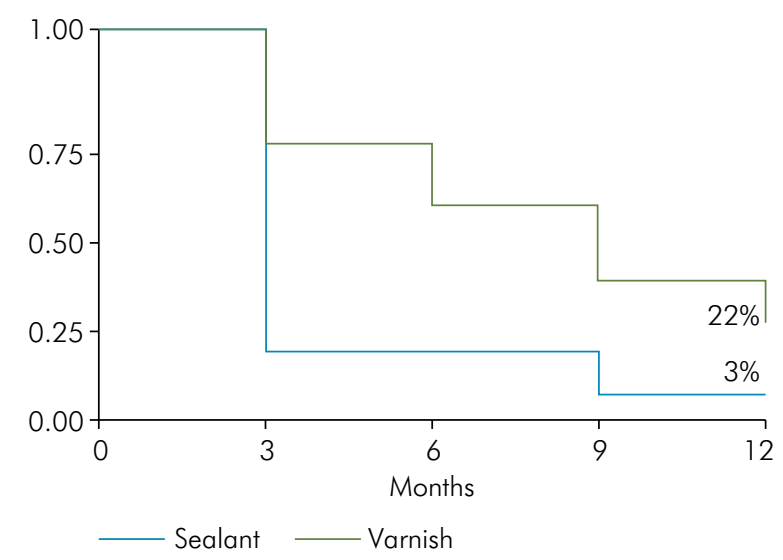

Figure 2. Kaplan-Meier survival curves showing the percentages of not arrested carious lesions according to treatments $(p=0.04)$.

\section{Discussion}

This is the first study to compare non-invasive and micro-invasive treatments for the arrest of occlusal caries lesions in the enamel of erupting molars. Early caries development in the occlusal surfaces as evidenced by the high number of active carious lesions observed in the newly erupted teeth. ${ }^{7,16}$ This leads to the need for implementing non-operative treatments that are capable of arresting such lesions to avoid caries progression and cavitation into dentin before the teeth reach full occlusion. Although the original carious lesion could not be assessed after sealant application and the clinical success-failure criteria were different in both groups, this study demonstrated that the resin-modified glass ionomer sealant was more effective than fluoride varnish in arresting the occlusal enamel carious lesions of erupting permanent molars. The probability of the active enamel carious lesions to arrest after sealant application was 8.85 times higher than that of fluoride varnish applications $(p=0.01)$. This result is in line with a previous study that concluded that unsealed occlusal surfaces with incipient carious lesions had a greater potential for developing dentin lesions than those sealed with the glass ionomer cement. ${ }^{17}$ Scientific literature has shown that pit and fissure sealants performed better than fluoride varnish in the prevention of dental decay on occlusal surfaces. ${ }^{18}$ 
Table 3. Unadjusted and adjusted Hazard Ratios ( $\mathrm{HR} ; 95 \% \mathrm{Cl})$ for arresting active enamel carious lesions in erupting permanent molars (Cox regression model).

\begin{tabular}{|c|c|c|c|c|}
\hline Variables & $\mathrm{HR}_{\text {crude }}(95 \% \mathrm{Cl})$ & $p$-value & $\mathrm{HR}_{\text {adiusted }}(95 \% \mathrm{Cl})$ & $p$-value \\
\hline Age (years) & $0.37(0.17-0.79)$ & 0.01 & $0.42(0.22-0.78)$ & 0.01 \\
\hline \multicolumn{5}{|l|}{ Gender } \\
\hline Male & 1 & & & \\
\hline Female & $3.67(0.50-26.73)$ & 0.30 & & \\
\hline \multicolumn{5}{|l|}{ Biofilm at arrestment } \\
\hline Absence & 1 & & & \\
\hline Presence & $0.68(0.28-1.66)$ & 0.40 & & \\
\hline \multicolumn{5}{|c|}{ Eruption stage at arrestment } \\
\hline Partial eruption & 1 & & & \\
\hline Full occlusion & $0.67(0.27-1.72)$ & 0.41 & & \\
\hline \multicolumn{5}{|l|}{ Treatment } \\
\hline Fluoride varnish & 1 & & 1 & \\
\hline Sealant & $6.97(1.05-46.16)$ & 0.04 & $8.85(1.57-49.78)$ & 0.01 \\
\hline
\end{tabular}

HR: hazard ratio; $\mathrm{Cl}$ : confidence interval.

The biofilm might play a critical role in this process. Thick plaques have been strongly associated with caries activity. ${ }^{16}$ However, in our study, biofilm reduction was not related to carious lesion arrest. This may be explained by the fact that sealed surfaces do not interact with biofilms, or do not go through cariogenic challenges. Therefore, sealed teeth would show better results in short periods. Moreover, all participants received special instructions on dietary habits and appropriate techniques for plaque control on the occlusal surfaces of erupting permanent molars.

The eruption stage was also not associated with the arrest of carious lesions. Most sealed lesions were arrested in the first 3 months of follow-up, irrespective of the eruption stage. This result may be explained by the capacity of the material to block the biofilm's direct contact with carious lesions, and thus, stopping caries progression in the early eruption stages. The sealant's success depends only on its retention rate. ${ }^{19}$ In our study, four sealants were partially lost, while one was completely lost after 12 months of follow-up; however, carious lesions remained active in only one of these molars. Although there is no consensus regarding the application of fluoride varnish, ${ }_{1}^{14}$ four applications at weekly intervals seem appropriate for biofilm control and thus, may be adequate for treating enamel active carious lesions in clinical practice. It was observed that the lesions treated with fluoride varnish were more likely to remain active for a longer period, arresting progressively as the tooth erupted and oral hygiene became easier to perform. After 12 months, 22\% of the lesions remained active, and most progressed to enamel breakdown (ICDAS score $=3$ ) despite fluoride varnish applications. Therefore, to avoid greater dental structural losses, these teeth were sealed. The high rate of treatment failure may be related to biofilm interaction, as aforementioned. The participant's age was also associated with the arrest of the enamel carious lesions. Moreover, an association between biofilm control and age has been demonstrated in the literature, where older children performed better mechanical biofilm removal than younger children. ${ }^{20}$ The ability to perform effective toothbrushing, depends not only on the patient's age, but also on dexterity, psychological development, and individual motivation. ${ }^{21}$ In our study, children up to the age of 7 or 8 years were supervised daily by a caregiver owing to their limited motor skills. This may have contributed to a higher probability of arresting active enamel carious lesions in younger children (HR 0.42, 95\%CI 0.22-0.78; $\mathrm{p}=0.01$ ).

According to long-term analysis, the arrest of enamel carious lesions in erupting molars was treatmentdependent. As observed, the sealant promoted faster arrest of carious lesions than the fluoride varnish applications. Overall caries control is also dependent on patient-level interventions, such as diet counseling ${ }^{22}$ and oral hygiene instructions and reinforcement ${ }^{8}$. These 
interventions require adequate patient compliance for success. Therefore, resin-glass ionomer cement sealant could be a good alternative for preventing cavitation in active carious lesions that are more likely to progress, especially in erupting molars of high-risk children owing to greater difficulty in controlling biofilm accumulation. By placing sealants, clinicians may also have more time to address the challenges of effectively modifying hygiene and dietary habits. Maintaining a dry field and using the proper application technique is essential for the effectiveness and retention of resin-based sealant. If maintaining a dry field is not possible, clinicians should consider other treatments, such as fluoride varnish applications, for controlling the associated etiological factors of caries. To conclude, resin-modified glass ionomer cement sealants performed better than fluoride varnish applications for arresting carious lesions in erupting permanent molars, after a 12-month follow-up period.

\section{Acknowledgments}

We thank the National Coordination of Post-Graduate Education (CAPES), 3M ESPE, and Colgate/Palmolive, Brazil for their support.

\section{References}

1. Gimenez T, Bispo BA, Souza DP, Viganó ME, Wanderley MT, Mendes FM, et al. Does the decline in caries prevalence of Latin American and Caribbean children continue in the new century? Evidence from systematic review with meta-analysis. PLoS One. 2016 Oct;11(10):e0164903. https://doi.org/10.1371/journal.pone.0164903

2. Mimoza C, Vito MA. The first permanent molar most affected by dental caries - A longitudinal study. Int J Dent Med. 2018;4(2):36-41.

3. Mejàre I, Axelsson S, Dahlén G, Espelid I, Norlund A, Tranæus S, et al. Caries risk assessment. A systematic review. Acta Odontol Scand. 2014 Feb;72(2):81-91. https://doi.org/10.3109/00016357.2013.822548

4. Ekstrand KR, Christiansen J, Christiansen ME. Time and duration of eruption of first and second permanent molars: a longitudinal investigation. Community Dent Oral Epidemiol. 2003 Oct;31(5):344-50. https://doi.org/10.1034/i.1600-0528.2003.00016.x

5. Alves LS, Zenkner JE, Wagner MB, Damé-Teixeira N, Susin C, Maltz M. Eruption stage of permanent molars and occlusal caries activity/arrest. J Dent Res. 2014 Jul;93(7 Suppl):114S-9S. https://doi.org/10.1177/0022034514537646

6. Maltz M, Barbachan e Silva B, Carvalho DQ, Volkweis A. Results after two years of non-operative treatment of occlusal surface in children with high caries prevalence. Braz Dent J. 2003;14(1):48-54. https://doi.org/10.1590/S0103-64402003000100009

7. Oliveira RS, Zenkner JE, Maltz M, Rodrigues JA. Effectiveness of a standardized treatment protocol for children with active non-cavitated occlusal lesions on erupting permanent molars. Int J Paediatr Dent. 2015 Nov;25(6):393-8. https://doi.org/10.1111/ipd.12141

8. Slayton RL, Urquhart O, Araujo MW, Fontana M, Guzmán-Armstrong S, Nascimento MM, et al. Evidence-based clinical practice guideline on nonrestorative treatments for carious lesions: A report from the American Dental Association. J Am Dent Assoc. 2018 Oct;149(10):837-849.e19. https://doi.org/10.1016/i.adaj.2018.07.002

9. Schulz KF, Altman DG, Moher D. CONSORT 2010 statement: updated guidelines for reporting parallel group randomised trials. Int J Surg. 2011;9(8):672-7. https://doi.org/10.1016/j.ijsu.2011.09.004

10. Bravo M, Montero J, Bravo JJ, Baca P, Llodra JC. Sealant and fluoride varnish in caries: a randomized trial. J Dent Res. 2005 Dec;84(12):1138-43. https://doi.org/10.1177/154405910508401209

11. Ekstrand KR, Gimenez T, Ferreira FR, Mendes FM, Braga MM. The International Caries Detection and Assessment System - ICDAS: A Systematic Review. Caries Res. 2018;52(5):406-19. https://doi.org/10.1159/000486429

12. Carvalho JC, Ekstrand KR, Thylstrup A. Dental plaque and caries on occlusal surfaces of first permanent molars in relation to stage of eruption. J Dent Res. 1989 May;68(5):773-9. https://doi.org/10.1177/00220345890680050401

13. Nyvad B, Baelum V. Nyvad Criteria for caries lesion activity and severity assessment: A validated approach for clinical management and research. Caries Res. 2018;52(5):397-405. https://doi.org/10.1159/000480522

14. Lenzi TL, Montagner AF, Soares FZ, Rocha RO. Are topical fluorides effective for treating incipient carious lesions?: A systematic review and meta-analysis. J Am Dent Assoc. 2016 Feb;147(2):84-91.e1. https://doi.org/10.1016/i.adaj.2015.06.018

15. Braga MM, Mendes FM, De Benedetto MS, Imparato JC. Effect of silver diammine fluoride on incipient caries lesions in erupting permanent first molars: a pilot study. J Dent Child (Chic). 2009 Jan-Apr;76(1):28-33.

16. Zenkner JE, Alves LS, de Oliveira RS, Bica RH, Wagner MB, Maltz M. Influence of eruption stage and biofilm accumulation on occlusal caries in permanent molars: a generalized estimating equations logistic approach. Caries Res. 2013;47(3):177-82. https://doi.org/10.1159/000345076 
17. Taifour D, Frencken JE, van't Hof MA, Beiruti N, Truin GJ. Effects of glass ionomer sealants in newly erupted first molars after 5 years: a pilot study. Community Dent Oral Epidemiol. 2003 Aug;31(4):314-9. https://doi.org/10.1034/i.1600-0528.2003.00039.x

18. Hiiri A, Ahovuo-Saloranta A, Nordblad A, Mäkelä M. Pit and fissure sealants versus fluoride varnishes for preventing dental decay in children and adolescents. In: Hiiri A, editor. Cochrane database of systematic reviews. Chichester: John Wiley \& Sons; 2010.

19. Ahovvo-Saloranta A, Forss H, Hiiri A, Nordblad A, Mäkelä M. Pit and fissure sealants versus fluoride varnishes for preventing dental decay in the permanent teeth of children and adolescents. Cochrane Database Syst Rev. 2016 Jan;(1):CD003067. https://doi.org/10.1002/14651858.CD003067.pub4

20. Sandström A, Cressey J, Stecksén-Blicks C. Tooth-brushing behaviour in 6-12 year olds. Int J Paediatr Dent. 2011 Jan;21(1):43-9. https://doi.org/10.1111/i.1365-263X.2010.01080.x

21. Das UM, Singhal P. Tooth brushing skills for the children aged 3-11 years. J Indian Soc Pedod Prev Dent. 2009 Apr-Jun;27(2):104-7. https://doi.org/10.4103/0970-4388.55335

22. Moynihan PJ, Kelly SA. Effect on caries of restricting sugars intake: systematic review to inform WHO guidelines. J Dent Res. 2014 Jan;93(1):8-18. https://doi.org/10.1177/0022034513508954 